\title{
A saúde no licenciamento ambiental: uma proposta metodológica para a avaliação dos impactos da indústria de petróleo e gás
}

\author{
Health and environmental licensing: a methodological proposal \\ for assessment of the impact of the oil and gas industry
}

Eduardo Macedo Barbosa ${ }^{1}$

Matha Macedo de Lima Barata ${ }^{2}$

Sandra de Souza Hacon ${ }^{3}$

${ }^{1}$ Petróleo Brasileiro S.A.

Abstract Bearing in mind the importance of the impacts of the oil industry on human health, this article seeks to present a methodological proposal for analysis of these aspects in environmental impact assessment studies, based on the established legal parameters and a validated matrix for the hydroelectric sector. The lack of health considerations in the environmental impact assessment was detected in most of the 21 oil production enterprises analyzed, that were licensed in the period from January 1, 2004 through October 30, 2009. The health matrix proved to be an appropriate methodological approach to analyze these aspects in the environmental licensing process, guiding decisions and interventions in socio-environmental management.

Key words Health impact assessment, Environmental management, Environmental licensing, Oil and gas industry
Resumo Considerando a importância dos impactos da indústria do petróleo sobre a saúde humana, este artigo apresenta uma proposta metodológica para a análise desses aspectos nos estudos de avaliação de impacto ambiental, a partir dos parâmetros legais estabelecidos e da adaptação de uma matriz validada para o setor hidrelétrico. A análise dos relatórios ambientais de 21 empreendimentos nacionais de produção de petróleo, licenciados no período de $1^{\circ}$ de janeiro de 2004 a 31 de outubro de 2009, constatou a inexistência de evidências da incorporação de aspectos de saúde na maioria dos seus respectivos estudos de avaliação de impacto ambiental. A matriz mostrou-se um instrumento metodológico adequado para analisar a inserção da saúde no licenciamento ambiental e orientar a tomada de decisão e intervenções para a gestão socioambiental.

Palavras-chave Avaliação de impactos à saúde, Gestão ambiental, Licenciamento ambiental, Indústria de petróleo e gássil
(Petrobras). Av. República do Chile 500, Centro. 20031-170 Rio de Janeiro RJ.eduardobarbosa@ petrobras.com.br

${ }^{2}$ Instituto Oswaldo Cruz,

Fundação Instituto Oswaldo Crus (Fiocruz/IOC)

${ }^{3}$ Escola Nacional de Saúde Pública, Fundação Instituto Oswaldo Cruz (Fiocruzl ENSP) 


\section{Introdução}

Durante muitos anos, os problemas e impactos ambientais decorrentes do desenvolvimento econômico foram considerados como um "mal necessário", justificados pelos benefícios proporcionados pelo progresso. Argumentava-se sobre a incompatibilidade entre a maximização de lucros e a responsabilidade socioambiental das empresas e entre a política ambiental e o crescimento da atividade econômica de um país. A economia baseava-se na maximização de lucros no curto prazo, em função do mercado de produtos e insumos e da reação à regulamentação governamental, e as empresas limitavam-se a cumprir as normas de poluição ambiental instituídas pelos órgãos reguladores e de controle ${ }^{1}$.

O crescimento econômico e o desenvolvimento tecnológico observados nas últimas décadas propiciaram uma crescente diversificação das atividades e produtos da indústria. A busca por novos modelos econômicos e tecnológicos diante das crescentes exigências e pressões da sociedade em relação à preservação ambiental, da forte concorrência num mercado cada vez mais globalizado e da preocupação dos acionistas com a saúde financeira de seus negócios impuseram um novo desafio para as empresas: serem competitivas e sustentáveis ao mesmo tempo ${ }^{2}$.

Não obstante a reestruturação da maioria das empresas frente a este desafio, os esforços nessa direção ainda encontram-se mais voltados para o ambiente interno de suas organizações e para os processos tecnológicos, produtos e práticas de gestão que não consideram os aspectos sociais e de saúde de uma forma integral, mantendo o foco na compensação dos impactos ao meio ambiente e no contexto filantrópico da dimensão social, numa abordagem reativa de gestão socioambiental ${ }^{3}$.

A complexidade dos problemas socioambientais e a múltipla determinação social da saúde, incluindo questões básicas como a moradia, o saneamento, o emprego, a renda, a educação e o acesso aos bens e serviços de saúde, impõem a necessidade de uma abordagem sistêmica, interdisciplinar e intersetorial para as questões do desenvolvimento, que permita apreender sua globalidade e as inter-relações causais entre seus impactos ambientais e respectivos efeitos à saúde humana, rompendo com o modelo clássico do processo saúde-doença ${ }^{4,5}$.

Os efeitos à saúde humana decorrentes dos impactos ambientais de um empreendimento deveriam ser considerados em todo o processo de licenciamento ambiental de suas atividades e operações. Mas na prática, esses aspectos são mal avaliados ou até mesmo nem são contemplados, pois apesar da Avaliação de Impacto Ambiental (AIA) levar em consideração os aspectos relativos ao ambiente físico, biótico, e socioeconômico, não explicita os potenciais impactos à saúde da população estabelecida em sua área de influência ${ }^{6}$. Entende-se como área de influência a área geográfica e as comunidades que podem ser direta ou indiretamente impactadas pelas atividades e operações de um determinado empreendimento. A caracterização da área de influência abrange os ecossistemas e os aspectos socioeconômicos, culturais e de saúde.

Os estudos de AIA, etapa necessária para determinar se o empreendimento é passível de ser licenciado, têm como objetivo prevenir as consequências e os danos ambientais decorrentes da instalação e operação de projetos e empreendimentos potencialmente poluidores, através da mensuração e comunicação de seus riscos, vantagens e desvantagens, assim como das alternativas e medidas de mitigação e compensação previstas.

Pesquisas e trabalhos na área de licenciamento e avaliação dos impactos ambientais têm identificado importantes desafios para a inserção dos impactos à saúde nos Estudos e nos Relatórios de Impacto Ambiental (EIA/RIMA) ${ }^{7,8}$. No setor de petróleo e gás natural, o Programa das Nações Unidas para o Meio Ambiente (PNUMA) no seu relatório "The Oil Sector Report: a Review of Environmental Disclosure in the Oil Industry", publicado em 1999, já evidenciava a importância da identificação e avaliação dos impactos à saúde além da simples quantificação dos poluentes ambientais, idealizando assim um processo integrado de gestão socioambiental proativo?.

No Brasil, apesar do licenciamento ambiental e da AIA, instrumentos da Política Nacional de Meio Ambiente, representarem um marco histórico para a prevenção e controle dos impactos ambientais decorrentes do desenvolvimento, ainda há a necessidade de estruturação sistemática da participação do setor saúde neste processo, através da criação de instrumentos que contribuam para induzir as empresas a mitigar e compensar seus impactos à saúde da população ${ }^{10}$.

Diante do atual contexto nacional desenvolvimentista, um exemplo de programa governamental responsável pela implantação de grandes empreendimentos que, de acordo com o porte e o tipo de atividade, podem impactar o meio ambiente e influenciar a saúde de diferentes grupos populacionais, é o Programa de Aceleração do 
Crescimento (PAC). Este programa tem como principal eixo a infraestrutura energética, com importantes investimentos em dois importantes segmentos: a geração e a transmissão de energia elétrica, e a produção, a exploração e o transporte de petróleo, gás natural e combustíveis renováveis. Não obstante, ele não pode estar dissociado do anseio global e nacional do desenvolvimento sustentável, que é ancorado no tripé: desenvolvimento econômico, justiça social e manutenção da qualidade dos ecossistemas.

Porto e Milanez ${ }^{11}$ chamam a atenção para a existência de eixos de desenvolvimento econômico ambientalmente insustentáveis, que intensificam os problemas socioambientais nacionais decorrentes de uma visão economicista restrita, representados pelo setor de produção de energia e as grandes obras de infraestrutura, exemplificados pelos derramamentos de óleo e derivados da indústria do petróleo e pelo desmatamento e degradação ambiental das barragens e usinas hidrelétricas. Apesar dos acidentes com vazamentos de petróleo não representarem a principal fonte de contaminação ambiental dos mares e oceanos, podem causar prejuízos socioeconômicos e importantes impactos ambientais e à saúde humana ${ }^{12-14}$.

Considerando a importância dos efeitos à saúde relacionados, direta e indiretamente, aos impactos ambientais de grandes empreendimentos voltados para a infraestrutura energética, esse artigo tem como objetivo apresentar uma proposta metodológica para a avaliação da incorporação da dimensão saúde no licenciamento ambiental do setor petróleo, considerando os impactos socioambientais e de saúde, a partir da adaptação da matriz de análise das questões de saúde, utilizada e validada por Cancio $^{15}$, para o setor hidrelétrico.

\section{Licenciamento Ambiental}

No Brasil, o licenciamento ambiental foi instituído pela Lei 6.938, de 31 de agosto de 1981, que introduziu a AIA para promover o controle prévio à instalação e à operação de empreendimentos que utilizem recursos ambientais, considerados potencialmente poluidores. Dentre as atividades que necessitam de licenciamento ambiental destacam-se as de extração e tratamento de minerais, as indústrias química e metalúrgica, e as atividades que utilizam recursos naturais ${ }^{16}$.

O licenciamento ambiental tem sua fundamentação legal na Resolução CONAMA 001, de 23 de janeiro de 1986, e na Resolução CONAMA
237, de 19 de dezembro de 1997, que estabeleceram a exigência de estudos ambientais voltados à identificação e avaliação de impactos desses empreendimentos, estruturados através do Estudo de Impacto Ambiental e do Relatório de Impacto Ambiental (EIA/RIMA), definidos por essas mesmas resoluções ${ }^{17,18}$.

A AIA determina os tipos de empreendimentos que dependerão da elaboração dos EIA/RIMA para a obtenção de licença ambiental, que deverão abordar, no mínimo, os seguintes aspectos:

- Diagnóstico ambiental da área de influência do projeto, contendo a descrição dos recursos ambientais e suas interações, caracterizando as condições ambientais antes da implantação do projeto, contemplando os meios físico, biótico e socioeconômico;

- Análise dos impactos ambientais do projeto e de suas alternativas, através de identificação, previsão da magnitude e interpretação da importância dos prováveis impactos relevantes (diretos e indiretos; imediatos e a médio e longo prazos; temporários e permanentes; e seu grau de reversibilidade e de distribuição dos ônus e benefícios sociais);

- Estudo de medidas mitigadoras destinadas a corrigir ou anular os impactos negativos ou a reduzir sua magnitude;

. Estabelecimento de programas de acompanhamento e monitoramento, de modo que se possam comparar, durante a implantação e operação da atividade, os impactos previstos com os que efetivamente ocorreram.

A AIA deve considerar os aspectos ambientais, socioeconômicos e de saúde, considerando diferentes alternativas tecnológicas e de localização do projeto, assim como a alternativa de não realização do mesmo, e propor medidas de mitigação e compensação dos seus respectivos impactos, numa abordagem de avaliação mais estratégica. Em geral, os estudos ambientais exigidos não consideram as interações do projeto proposto com outras atividades antrópicas existentes, nem com políticas, planos e programas de governo ${ }^{15,19}$.

A competência para o licenciamento ambiental leva em consideração a abrangência e o grau de impacto, podendo ser classificado em intercontinental, nacional, regional, intermunicipal e local. Na esfera federal, compete ao Instituto Brasileiro de Meio Ambiente e dos Recursos Naturais Renováveis (IBAMA) o licenciamento de atividades de âmbito nacional ou regional, localizados ou desenvolvidos em dois ou mais Estados, no mar territorial e na plataforma continental, 
cujos impactos ambientais diretos ultrapassem esses limites territoriais. O IBAMA autorizará o licenciamento ambiental após avaliação técnica dos órgãos ambientais dos Estados e Municípios em que se localizar o empreendimento, podendo considerar ainda a anuência de demais órgãos competentes da União, Estados e Municípios, como os de saúde. O IBAMA poderá também delegar aos Estados o licenciamento de atividade com significativo impacto ambiental de âmbito regional, uniformizando, quando possível, as exigências necessárias.

Para o licenciamento ambiental dos empreendimentos da indústria de petróleo, gás natural e derivados, os órgãos ambientais consideram a necessidade da elaboração de um diagnóstico ambiental contemplando o meio físico (geologia, geomorfologia, meteorologia, oceanografia, qua- lidade do ar, da água e do solo, e a dispersão de emissões atmosféricas e de óleo e efluentes), o meio biótico e o meio socioeconômico. Os impactos ambientais gerados por esse setor sempre foram mais tolerados, considerando a justificativa dessa atividade ser fundamental para o modelo desenvolvimentista e de sua importância para a matriz energética mundial (Quadro 1) ) $^{19-21}$.

Entretanto, o crescimento do setor petróleo e o papel estratégico do meio ambiente, perante tal atividade, exigem ações que privilegiem a articulação intersetorial para a implementação de um processo de gestão ambiental cada vez mais integrado às questões sociais e de saúde.

O licenciamento ambiental das atividades de exploração e produção marítimas de petróleo e gás natural é também de responsabilidade do IBAMA, através da Coordenação Geral de Licen-

Quadro 1. Impactos socioambientais e à saúde humana nas diferentes áreas do setor petróleo

\begin{tabular}{|c|c|c|}
\hline $\begin{array}{l}\text { Áreas do setor } \\
\text { petróleo }\end{array}$ & Ações e efeitos diretos & Impactos socioambientais e à saúde \\
\hline Exploração "on shore" & Desmatamento & Doenças infecciosas emergentes \\
\hline \multirow{4}{*}{$\begin{array}{l}\text { Perfuração e } \\
\text { Produção }\end{array}$} & $\begin{array}{l}\text { Descargas de hidrocarbonetos e } \\
\text { fluidos de perfuração }\end{array}$ & $\begin{array}{l}\text { Degradação ambiental crônica, com } \\
\text { contaminação química e física }\left(\mathrm{NORM}^{*}\right)\end{array}$ \\
\hline & $\begin{array}{l}\text { Ruído e disposição de oleodutos } \\
\text { sobre estuários }\end{array}$ & $\begin{array}{l}\text { Modificações do habitat natural de animais, } \\
\text { com ilhas e recifes artificiais }\end{array}$ \\
\hline & Contaminação do meio físico & $\begin{array}{l}\text { Redução da pesca e do gado, aumento da } \\
\text { morbidade e mortalidade de aves, animais } \\
\text { marinhos e domésticos, comprometimento } \\
\text { da qualidade do ar, dos solos e das águas. }\end{array}$ \\
\hline & $\begin{array}{l}\text { Riscos para a saúde dos } \\
\text { trabalhadores e das comunidades }\end{array}$ & $\begin{array}{l}\text { Doenças cutâneas, respiratórias, mentais e } \\
\text { câncer }\end{array}$ \\
\hline \multirow[b]{2}{*}{ Transporte } & Poluição química e sonora & Danos aos ecossistemas \\
\hline & Derramamentos & $\begin{array}{l}\text { Destruição de vegetações, lavouras e animais } \\
\text { terrestres e litorâneos, contaminação de } \\
\text { lençóis freáticos. }\end{array}$ \\
\hline \multirow{2}{*}{ Refino } & $\begin{array}{l}\text { Exposição a substâncias e } \\
\text { materiais perigosos }\end{array}$ & $\begin{array}{l}\text { Doenças crônicas pulmonares e mentais e } \\
\text { neoplasias }\end{array}$ \\
\hline & $\begin{array}{l}\text { Riscos de incêndios, explosões, } \\
\text { vazamentos e derramamentos }\end{array}$ & Acidentes ambientais e ocupacionais \\
\hline \multirow{2}{*}{ Combustão } & $\begin{array}{l}\text { Emissão de particulados e } \\
\text { ozônio (poluição do ar) }\end{array}$ & $\begin{array}{l}\text { Mortes prematuras e doenças respiratórias e } \\
\text { cardiovasculares }\end{array}$ \\
\hline & $\begin{array}{l}\text { Emissão de } \mathrm{NOx}, \mathrm{SOx} \text { e } \mathrm{CO}_{2}{ }^{* *} \\
\text { (mudança climática) }\end{array}$ & $\begin{array}{l}\text { Chuva ácida, acidificação do solo, elevação da } \\
\text { temperatura e aquecimento global, impactos } \\
\text { na agricultura e saúde }\end{array}$ \\
\hline
\end{tabular}

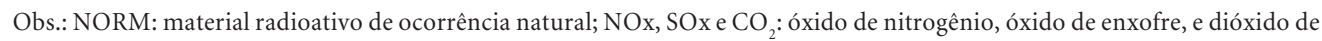
carbono, respectivamente. 
ciamento de Petróleo e Gás (CGPEG), cujos procedimentos estão regulamentados pelas Resoluções CONAMA 237/97, 23/94 e 350/04, que consideram como atividade de exploração e lavra de jazidas de combustíveis líquidos e gás natural o levantamento de dados sísmicos, a perfuração de poços para identificação das jazidas e suas extensões; a produção para pesquisa sobre a viabilidade econômica; e a produção efetiva para fins comerciais ${ }^{18,22,23}$.

\section{Licenciamento Ambiental e Saúde}

Apesar da definição de impacto ambiental da Resolução CONAMA no 1/86 fazer referência aos aspectos de saúde, e da Resolução CONAMA no 237/97 estabelecer direitos ao órgão ambiental de suspender ou cancelar uma licença em caso de ocorrência de graves riscos ambientais e de saúde, na prática, os estudos e relatórios exigidos se preocupam apenas com os impactos diretos ao meio ambiente. Os impactos sobre a saúde não são explicitados, pois ainda são considerados apenas como consequências indiretas dos impactos ao meio ambiente.

Apesar da relevância dos impactos à saúde decorrentes das atividades e operações industriais, os órgãos ambientais responsáveis pelo processo de licenciamento ambiental não estão preparados para analisar esses aspectos, havendo ainda limitações e dificuldades institucionais de gestão, infraestrutura e de natureza técnica para o estabelecimento de um processo integrado de vigilância e controle dos aspectos de ambiente e saúde ${ }^{24,25}$.

No Brasil, com a criação da Coordenação Geral de Vigilância Ambiental em Saúde (CGVAM) em 1999, foi instituído o Subsistema Nacional de Vigilância em Saúde Ambiental (SINVSA) como ferramenta de vigilância em saúde do Sistema Único de Saúde (SUS), cuja principal atribuição é prevenir e controlar os agravos à saúde das populações expostas aos impactos de empreendimentos poluidores. Para a estruturação e operacionalização de políticas e ações integradas de saúde e meio ambiente, o Ministério da Saúde institui, em 2001, um Termo de Cooperação Técnica com o Ministério do Meio Ambiente para a implementação de uma agenda nacional de Saúde Ambiental, com a identificação de áreas prioritárias de cooperação. E, mais recentemente, foi assinada a Portaria Interministerial 882, de 30 de abril de 2008, entre os Ministérios da Saúde e do Meio Ambiente, estabelecendo diretrizes para a integração e cooperação através da implementação de ações comuns e consolidação de agenda bilateral ${ }^{10,26}$.

$\mathrm{Na}$ tentativa de estabelecer parâmetros de avaliação da relação entre impacto ambiental e impacto à saúde, o Ministério da Saúde já identificou a necessidade de normatização específica para estruturar a participação do setor saúde nos processos de licenciamento ambiental de empreendimentos que causem riscos à saúde humana. Além da intenção de inserção de aspectos de saúde nos termos de referência, nos estudos e relatórios de impacto ambiental, e nos demais documentos pertinentes ao processo de emissão das licenças ambientais, há também recomendações de ações para a prevenção e o controle dos fatores de risco à saúde das populações das áreas de influência desses empreendimentos ${ }^{27}$.

Atualmente, os únicos mecanismos institucionais de participação da saúde no processo de licenciamento ambiental se dão através da Resolução CONAMA 286/2001, que regulamenta o licenciamento de empreendimentos em regiões endêmicas de malária ${ }^{28}$, e da Portaria 47/2007, da Secretaria de Vigilância em Saúde do Ministério da Saúde (SVS/MS), que dispõe sobre a Avaliação do Potencial Malarígeno e o Atestado de Condição Sanitária para projetos de assentamento de reforma agrária e outros empreendimentos nessas regiões ${ }^{10}$.

\section{Metodologia}

A escolha de empreendimentos do setor petróleo cadastrados no PAC considerou sua representatividade e importância em relação ao desenvolvimento do país. Com o objetivo de instrumentalizar a avaliação dos impactos desse setor sobre a saúde, aplicou-se uma matriz de análise da inserção de aspectos de saúde nos estudos e nos relatórios de impacto ambiental referentes ao licenciamento de atividades de produção de petróleo. Essa matriz foi elaborada a partir da matriz utilizada e validada por Cancio ${ }^{15}$ para o setor hidrelétrico, considerando sua similaridade com a indústria de petróleo, em relação ao potencial poluidor e seus respectivos impactos e às categorias analíticas e a aspectos de saúde selecionados com base na legislação ambiental vigente.

Nessa matriz, Cancio ${ }^{15}$ partiu de uma abordagem qualitativa, fundamentada na interpretação de fenômenos e atribuições de significados, buscando alcançar a unidade na diversidade e produzir explicações, comportando vários graus de generalização e aproximação ao objeto da pes- 
quisa. A classificação dos aspectos de saúde em diferentes grupos de categorias analíticas e sua respectiva organização em categorias operacionais serve como guia teórico e de balizamento para o conhecimento do objeto de pesquisa, permitindo a observação e o trabalho de campo ${ }^{29}$.

Para a inclusão dos EIA/RIMA nesta pesquisa foram utilizadas as informações disponíveis no banco de dados do Sistema Informatizado de Licenciamento Ambiental Federal (SISLIC) do IBAMA $^{30}$, considerando-se os seguintes critérios para a seleção dos empreendimentos:

- estar na competência de licenciamento da CGPEG;

. ter sido licenciado no período de $1^{\circ}$ de janeiro de 2004 a 31 de outubro de 2009;

. estar classificado na tipologia "Petróleo-Produção" e cadastrado no PAC.

Foram selecionados um total de 21 empreendimentos de produção de petróleo, apresentados no Quadro 1, cujos respectivos EIA/RIMA foram analisados em relação à inserção de aspectos e impactos à saúde humana, de acordo com as categorias consideradas na matriz proposta. Na avaliação da inserção dos aspectos de saúde nos estudos e relatórios selecionados, cada aspecto de saúde foi analisado segundo o grau de explicitação no EIA/RIMA como inexistente, parcial ou total.

A definição dos aspectos de saúde que compuseram as categorias operacionais e analíticas da matriz elaborada para a avaliação dos EIA/ RIMA no setor petróleo considerou os parâmetros de avaliação de impacto ambiental estabelecidos pela Resolução CONAMA 001/86. Os aspectos de saúde analisados foram agrupados em sete diferentes categorias: descrição do projeto; diagnóstico socioambiental das áreas de influência; identificação, análise e comunicação de impactos; compatibilidade entre ações do governo e empreendimento; avaliação de alternativas; identificação de medidas mitigadoras; e monitoramento e controle dos impactos à saúde.

$\mathrm{Na}$ "descrição do projeto" considerou-se a participação de profissionais qualificados em saúde na equipe técnica; a ocupação e o uso do solo; e as estimativas de geração de emprego e renda, assim como a aplicação dos recursos financeiros na melhoria da saúde e bem estar da população.

O "diagnóstico socioambiental das áreas de influência" foi analisado a partir do levantamento de aspectos epidemiológicos, socioeconômicos e da percepção dos impactos e riscos à saúde dessa população, com base em dados primários ou secundários. Nessa categoria, considerou-se também a respectiva infraestrutura de saúde e educação disponíveis.

A categoria "identificação, análise e comunicação de impactos" considerou a análise propriamente dita dos impactos à saúde humana, tanto de trabalhadores quanto da população das áreas de influência. A repercussão desses impactos sobre os indicadores socioeconômicos e de saúde dessas populações também foi considerada, com base nos conceitos e definições de indicadores de saúde da Rede Interagencial de Informações para a Saúde (RIPSA) ${ }^{31}$.

Em relação à "avaliação de alternativas" foram consideradas as locacionais, as tecnológicas e as econômicas, subsidiadas por uma análise de custo e benefício dos potenciais impactos socioambientais e de saúde relacionados ao tipo de empreendimento em questão.

Para a "identificação de medidas mitigadoras" a serem previstas pelos empreendedores, considerou-se os aspectos de saúde relacionados aos impactos do empreendimento propriamente dito, à infraestrutura e aos serviços de saúde disponíveis, e à vulnerabilidade social. A vulnerabilidade compreende um conjunto de aspectos além do individual, abrangendo questões coletivas e contextuais, que levam à suscetibilidade a doenças, considerando ainda a falta de recursos disponíveis para a proteção humana, reforçando a responsabilização da sociedade ${ }^{32}$.

Nessa categoria foi contemplada também a definição de planos de emergência, conforme o tipo e a abrangência dos impactos negativos identificados, como medida mitigadora dos mesmos.

$\mathrm{O}$ "monitoramento e controle dos impactos à saúde", relacionados à produção de petróleo, consideraram a definição de metas e indicadores de gestão dos impactos e riscos à saúde ocupacional e das comunidades da área de influência das respectivas atividades industriais.

\section{Resultados}

Os resultados quantitativos dessa pesquisa foram consolidados na matriz de análise apresentada na Tabela 1, e os aspectos de saúde evidenciados nos EIA/RIMA agrupados por categorias.

\section{Descrição do Projeto}

Na descrição e apresentação da equipe técnica responsável pela elaboração desses EIA/RIMA não evidenciamos a participação de quaisquer técni- 
Tabela 1. Matriz de análise dos aspectos de saúde nos EIA/RIMA dos empreendimentos do setor de produção de petróleo, cadastrados no PAC e licenciados de 01/01/2004 a 30/10/2009.

\begin{tabular}{|c|c|c|c|c|}
\hline \multirow[b]{2}{*}{ Categoria Analítica } & \multirow[b]{2}{*}{ Aspectos de Saúde } & \multicolumn{3}{|c|}{ Explicitação nos EIA/RIMA } \\
\hline & & Inexistente & Parcial & Total \\
\hline \multirow[t]{4}{*}{ Descrição do Projeto } & Equipe técnica responsável pela abordagem de saúde & 24 & & \\
\hline & $\begin{array}{l}\text { Dinâmica de ocupação e uso do território (solo, } \\
\text { água, e migrações e deslocamentos populacionais) }\end{array}$ & 10 & 14 & \\
\hline & $\begin{array}{l}\text { Estimativas quantitativas de geração de renda, } \\
\text { emprego, impostos e royalties }\end{array}$ & & 21 & 3 \\
\hline & $\begin{array}{l}\text { Estimativas de aplicação de recursos financeiros, } \\
\text { direta ou indiretamente, na melhoria da saúde }\end{array}$ & 16 & 8 & \\
\hline Diagnóstico & Perfil epidemiológico & 20 & 4 & \\
\hline Socioambiental das & Perfil socioeconômico & 17 & 7 & \\
\hline \multirow{3}{*}{ Áreas de Influência } & Recursos, serviços e infraestrutura de saúde & 21 & 3 & \\
\hline & Recursos, serviços e infraestrutura de educação & 21 & 3 & \\
\hline & Percepção dos impactos e riscos à saúde & 21 & 3 & \\
\hline \multirow{5}{*}{$\begin{array}{l}\text { Identificação, } \\
\text { Avaliação e } \\
\text { Comunicação de } \\
\text { Impactos }\end{array}$} & $\begin{array}{l}\text { Impactos e riscos à saúde dos trabalhadores } \\
\text { (físicos, químicos, ergonômicos e biológicos) }\end{array}$ & 14 & 10 & \\
\hline & Impactos e riscos à saúde da população & 24 & & \\
\hline & $\begin{array}{l}\text { Impacto do empreendimento sobre indicadores } \\
\text { de saúde (morbidade, mortalidade, atendimentos } \\
\text { ambulatorial e hospitalar, etc.) }\end{array}$ & 24 & & \\
\hline & $\begin{array}{l}\text { Impacto do empreendimento sobre indicadores } \\
\text { sociais e econômicos (PIB, escolaridade, } \\
\text { saneamento, emprego e renda, etc.) }\end{array}$ & 5 & 19 & \\
\hline & Comunicação dos impactos à saúde & 3 & 21 & \\
\hline \multirow{4}{*}{$\begin{array}{l}\text { Compatibilidade } \\
\text { entre ações do } \\
\text { governo e } \\
\text { empreendimento }\end{array}$} & $\begin{array}{l}\text { Identificação de planos, projetos e programas do } \\
\text { governo }\end{array}$ & 16 & 3 & 5 \\
\hline & $\begin{array}{l}\text { Avaliação da compatibilidade entre ações } \\
\text { governo e empreendimento }\end{array}$ & 17 & 7 & \\
\hline & Avaliação das ações do governo sobre a saúde & 21 & 3 & \\
\hline & $\begin{array}{l}\text { Avaliação das ações do empreendimento sobre a } \\
\text { saúde }\end{array}$ & 21 & 3 & \\
\hline \multirow{4}{*}{$\begin{array}{l}\text { Avaliação de } \\
\text { alternativas }\end{array}$} & Avaliação de alternativas locacionais & 16 & 4 & 4 \\
\hline & Avaliação de alternativas tecnológicas & 14 & 7 & 3 \\
\hline & Avaliação de alternativas econômicas & 10 & 14 & \\
\hline & $\begin{array}{l}\text { Análise de custo-benefício dos potenciais } \\
\text { impactos à saúde }\end{array}$ & 24 & & \\
\hline \multirow[t]{3}{*}{$\begin{array}{l}\text { Identificação de } \\
\text { Medidas Mitigadoras }\end{array}$} & $\begin{array}{l}\text { Medidas mitigadoras referentes aos impactos do } \\
\text { empreendimento }\end{array}$ & 21 & 3 & \\
\hline & $\begin{array}{l}\text { Medidas mitigadoras referentes à infraestrutura e } \\
\text { aos serviços de saúde }\end{array}$ & 24 & & \\
\hline & $\begin{array}{l}\text { Medidas mitigadoras específicas referentes às } \\
\text { populações sob risco e mais vulneráveis } \\
\text { Definição de planos de emergência e } \\
\text { contingência considerando o tipo e a } \\
\text { abrangência dos impactos }\end{array}$ & 24 & 21 & 3 \\
\hline \multirow{4}{*}{$\begin{array}{l}\text { Monitoramento e } \\
\text { controle dos } \\
\text { impactos à saúde }\end{array}$} & $\begin{array}{l}\text { Acompanhamento de ações de saúde do } \\
\text { trabalhador pelo empreendimento }\end{array}$ & & 17 & 7 \\
\hline & $\begin{array}{l}\text { Acompanhamento de ações de saúde da } \\
\text { população pelo empreendimento }\end{array}$ & 24 & & \\
\hline & $\begin{array}{l}\text { Acompanhamento das ações de saúde do } \\
\text { trabalhador pelo governo }\end{array}$ & 24 & & \\
\hline & $\begin{array}{l}\text { Acompanhamento das ações de saúde da } \\
\text { população pelo governo }\end{array}$ & 24 & & \\
\hline
\end{tabular}


cos da área saúde em nenhum dos estudos e relatórios analisados. Portanto, os possíveis aspectos e impactos de saúde a serem contemplados não foram identificados e, muito menos, avaliados por profissionais com qualificação técnica específica em saúde em 100\% dos estudos analisados, comprometendo a realização de uma abordagem integrada, interdisciplinar e sistêmica.

Em relação à avaliação da dinâmica de ocupação e uso do território, relativo ao aproveitamento do solo e água, além de migrações e deslocamentos populacionais, observou-se que em 57\% dos EIA/RIMA, ou seja, 12 estudos analisados, consideraram parcialmente esses aspectos, enquanto que em 43\%, 9 estudos, não apresentaram quaisquer evidências para tal avaliação.

Constatamos que a maioria dos estudos da tipologia produção de petróleo, 86\%, representada por 18 estudos, apresentou estimativas quantitativas parciais de geração de renda, emprego, tributos e impostos como royalties. Entretanto, apenas 7 estudos explicitaram, de forma parcial, a previsão da aplicação desses recursos, direta ou indiretamente, na melhoria da saúde humana.

\section{Diagnóstico Socioambiental das Áreas de Influência}

Nessa categoria, evidenciamos que somente 30\% dos EIA/RIMA analisados, ou seja, 6 estudos explicitaram parcialmente o perfil socioeconômico das comunidades de suas respectivas área de influência, menos de $17 \%$ consideraram o levantamento epidemiológico, e apenas 14\% a percepção dos impactos e riscos à saúde, também de forma parcial, representados por 4 e 3 estudos, respectivamente.

O levantamento de recursos, serviços e infraestrutura de saúde e educação, pública e privada, das áreas de influência desses empreendimentos, e a percepção dos impactos e riscos à saúde de suas respectivas comunidades também foi considerado parcialmente em apenas 14\% dos estudos.

A análise dos aspectos de saúde considerados nessa categoria subsidiou a caracterização do grau de vulnerabilidade e risco à saúde de comunidades da área de influência do empreendimento. A identificação das populações vulneráveis e sob risco à saúde foi explicitada parcialmente em apenas 4 EIA/RIMA, que corresponde a menos de $17 \%$ dos estudos analisados.

\section{Identificação, Análise \\ e Comunicação de Impactos}

Em relação à identificação e avaliação dos impactos à saúde dos trabalhadores, 9 EIA/RIMA levantaram parcialmente os potenciais impactos e riscos da tipologia "petróleo-produção", que representou 43\% dos estudos. Quanto à população da área de influência, nenhum dos estudos analisados contemplou a avaliação integral dos impactos e riscos sobre a saúde dessas comunidades, com exceção para a comunicação dos riscos relacionados aos cenários de emergência que foi contemplada em $86 \%$ dos estudos.

Nenhum dos EIA/RIMA avaliou os impactos do empreendimento sobre os principais indicadores públicos de saúde, como morbidade, mortalidade e atendimento ambulatorial e hospitalar. Ao contrário, os indicadores sociais e econômicos foram avaliados, mesmo que parcialmente, em 17 estudos, representando 79\% destes.

\section{Compatibilidade entre ações do governo e empreendimento}

Em 14 estudos, que corresponderam a 65\% dos EIA/RIMA analisados, não foram identificados os planos, projetos e programas governamentais relacionados à saúde da população que poderiam ser compatíveis com os potenciais benefícios dos empreendimentos. Somente 21\% dos estudos explicitaram ações totalmente compatíveis com planos, projetos e programas do governo. Apenas $14 \%$ consideraram parcialmente a compatibilidade entre essas mesmas ações.

Em relação à avaliação da compatibilidade entre as ações de saúde do governo com as do empreendimento, 15 estudos, ou seja, 71\%, não consideraram tal compatibilidade, enquanto somente $6(29 \%)$ demonstraram-na, mesmo que não totalmente. Quanto às ações do governo e do empreendimento sobre a saúde humana, somente $14 \%$ dos estudos as consideraram parcialmente.

\section{Avaliação de alternativas}

Quanto às alternativas locacionais, aproximadamente $62 \%$ dos estudos analisados não consideraram tais alternativas, enquanto $38 \%$ explicitaram a necessidade dessa avaliação, sendo que apenas 4 estudos de forma total e outros 
4, parcialmente. Em relação às alternativas tecnológicas, apenas 6 estudos, ou seja 29\%, consideraram parcialmente a avaliação dessas alternativas, 3 totalmente, e os demais 12 estudos não a consideraram.

As alternativas econômicas foram consideradas em 12 estudos analisados, representando $57 \%$ dos mesmos. Enquanto os 9 estudos restantes não explicitaram tal alternativa.

Todos os estudos avaliados, que consideraram tais alternativas, foram subsidiados por análises de custo-benefício relacionadas somente aos impactos ambientais, e em nenhum deles explicitou-se qualquer consideração aos custos e benefícios em relação aos impactos à saúde.

\section{Identificação de Medidas mitigadoras}

Em nenhum dos estudos analisados, a identificação de medidas mitigadoras referentes aos impactos relacionados à infraestrutura e aos serviços de saúde, e especificamente aquelas referentes às populações mais vulneráveis e sob risco à saúde humana, foi considerada. Quanto à identificação de medidas mitigadoras relacionadas aos impactos do empreendimento, apenas 3 estudos, isto é, $14 \%$, consideraram tal aspecto. A definição de planos de emergência e contingência foi contemplada em todos os EIA/RIMA analisados, sendo que 18 estudos, isto é, $86 \%$ desses, consideraram esse aspecto de forma parcial.

\section{Monitoramento \\ e Controle dos Impactos à Saúde}

Nenhum dos estudos analisados explicitou a sistemática de acompanhamento e monitoramento das ações governamentais de saúde voltadas ao controle dos impactos do empreendimento sobre os trabalhadores e as comunidades das áreas de influência, bem como aquelas adotadas pelo empreendimento direcionadas às comunidades.

Entretanto, em relação às ações empresariais de controle dos impactos e riscos à saúde do trabalhador, 100\% dos EIA/RIMA explicitaram esse aspecto, sendo que somente 6 estudos, isto é, 29\%, definiram explicitamente as metas e os indicadores para o controle desses impactos, enquanto a grande maioria, $71 \%$, considerou essa sistemática apenas parcialmente.

\section{Discussão}

Apesar do esforço do Ministério do Meio Ambiente para garantir a sustentabilidade ambiental dos empreendimentos do PAC, os resultados obtidos com essa pesquisa confirmaram que os estudos e as avaliações de impactos ambientais não contemplam a saúde de forma sistemática, integral e harmonizada com os princípios da promoção da saúde e da sustentabilidade socioambiental. Demonstra-se, portanto, a necessidade da incorporação dos aspectos de saúde decorrentes dos impactos ambientais do setor petróleo e gás natural nos estudos de avaliação ambiental, subsidiando a definição de diretrizes e regulamentações para a melhoria do desempenho da gestão socioambiental deste setor.

Apesar da importância da identificação e caracterização de populações vulneráveis nas áreas de influência dos empreendimentos, considerando a multiplicidade dos fatores determinantes e condicionantes da saúde, observou-se que este aspecto precisa ser aprofundado metodologicamente e discutido politicamente para que seja incorporado aos estudos e relatórios de impacto ambiental, através de normatizações e legislações especificas.

A avaliação dos impactos à saúde humana, integrados aos estudos ambientais, poderá fornecer informações sobre as consequências e os efeitos na qualidade de vida, na saúde e no bem estar das pessoas, decorrentes das pressões exercidas pelas atividades industriais sobre o meio ambiente, ampliando a discussão sobre a desigualdade entre grupos sociais nas áreas de influência de seus empreendimentos. Os estudos de potenciais impactos negativos à saúde humana decorrentes das atividades, processos e produtos do setor petróleo podem contribuir para a minimização de seus efeitos e agravos, considerando o tipo e a abrangência do impacto ambiental. Entretanto, é necessário compartilhar decisões politicas, econômicas, sociais e ambientais para que os EIA/RIMA contemplem as vigilâncias epidemiológica e em saúde ambiental e do trabalhador integradas ao território onde o empreendimento será estabelecido.

Os técnicos das áreas de saúde e meio ambiente ainda não se encontram instrumentalizados e capacitados para uma abordagem integrada de 
Saúde e Ambiente, que pressupõe a adoção de ações interdisciplinares e intersetoriais mais articuladas, e uma maior integração da política ambiental com as políticas sociais, econômicas e de saúde.

Considerando a situação atual do setor petróleo, que se encontra em franca expansão com a introdução de novas formas de produção mais limpas e fontes renováveis de energia, acreditamos que a matriz composta por suas categorias analíticas e respectivos aspectos de saúde possa instrumentalizar a incorporação da avaliação dos impactos à saúde, tanto positivos quanto negativos, decorrentes de projetos de investimento na produção nacional de petróleo e gás natural, e orientar a tomada de decisão e intervenções no sentido de minimizar os efeitos negativos e maximizar os positivos sobre a saúde humana.

Adicionalmente a esse instrumento, acreditamos que outras técnicas e métodos como a Avaliação de Impacto à Saúde (AIS), a Avaliação de Risco à Saúde Humana (ARSH) e a Avaliação Ambiental Estratégica (AAE), poderão contribuir para a inserção dos aspectos de saúde no processo de licenciamento ambiental, propiciando uma atuação mais proativa e preventiva da gestão socioambiental em direção ao desenvolvimento sustentável, e contribuindo para o estabelecimento de um novo marco regulatório para a política nacional de saúde ambiental.

\section{Colaboradores}

MML Barata e SS Hacon participaram como orientadora e co-orientadora, respectivamente, da tese de doutorado de EM Barbosa, do qual resulta este artigo.

\section{Agradecimentos}

A Anderson Américo Alves Cantarino (D.Sc.) pelas importantes sugestões na revisão do artigo. 


\section{Referências}

1. Maimon D. A economia e a problemática ambiental. In: Vieira PF, Maimon D, organizadores. As ciências sociais e a questão ambiental: rumo à interdisciplinaridade. Belém: Universidade Federal do Pará (UFPa), Associação Portuguesa para estudo da Dor (APED); 1993.

2. Puppim de Oliveira JA. Empresas na Sociedade: Sustentabilidade e Responsabilidade Social. Rio de Janeiro: Editora Elsevier; 2008.

3. Faulkner D, Carlisle YM, Viney, HP. Changing corporate attitudes towards environmental policy. Manag Environ Qual Int J 2005; 16(5):476-489.

4. Gurgel AM, Medeiros ACLV, Alves PC, Silva JM, Gurgel IGD, Augusto, LGS. Framework dos cenários de risco no complexo da implantação de uma refinaria de petróleo em Pernambuco. Cien Saude Colet 2009; 14(6):2027-2038.

5. Silva JM, Gurgel AM, Gurgel IGD, Augusto LGS. Saúde: um tema ausente do licenciamento ambiental. In: Augusto LGS, organizadora. Saúde do trabalhador e sustentabilidade do desenvolvimento humano - ensaios em Pernambuco. Recife: Editora Universitária da UFPE; 2009. p. 313-329.

6. World Health Organization (WHO). Health Impact Assessment as part of Strategic Environmental Assessment. Geneva: World Health Organization (WHO), Regional Office for Europe; 2001.

7. Rigotto RM. Inserção da saúde nos estudos de impacto ambiental: o caso de uma termelétrica a carvão mineral no Ceará. Cien Saude Colet 2009; 14(6): 2049-2059.

8. Wernham A. Inupiat Health and Proposed Alaskan Oil Development: results of the First Integrated Health Impact Assessment/Environmental Impact Statement for Proposed Oil Development on Alaska's North Slope. EcoHealth 2007; 4(4):500-513.

9. United Nations Environment Program (UNEP). The Oil Sector Report. A Review of Environmental Disclosure in the Oil Industry. United Nations Environment Program (UNEP)/SustainAbility's Engaging Stakeholders Series. London: UNEP, SustainAbility Ltd.; 1999.

10. Silveira MA. Contribuição do Setor Saúde aos Processos de Licenciamento Ambiental de Empreendimentos: primeiras aproximações [dissertação]. Brasília: Universidade de Brasília; 2008.

11. Porto MF, Milanez B. Eixos de desenvolvimento econômico e geração de conflitos socioambientais no Brasil: desafios para a sustentabilidade e a justiça social. Cien Saude Colet 2009; 14(6):1983-1994.

12. United States Environmental Protection Agency. Understanding Oil Spills and Oil Spills Response. Washington-DC: USEPA; 1999. [Documento da Internet]. [acessado $2010 \mathrm{fev} 20$ ]. Disponível em: http:// www.epa.gov/oem/content/learning/pdfbook.htm.

13. Aleixo LAG, Tachibana T, Casagrande D. Poluição por óleo - Fontes de introdução de petróleo e derivados no ambiente. Integração 2007; 49:159-166.

14. Coastal Response Research Center. Environmental Performance Metrics for Oil Spill Response. Durham, New Hampshire: CRRC; 2006.
15. Cancio JA. Inserção das questões de saúde no estudo de impacto ambiental [dissertação]. Brasília-DF: Universidade Católica de Brasília; 2008.

16. Brasil. Lei $n^{\circ} 6.938$ de 31 de agosto de 1981. Dispõe sobre a Política Nacional do Meio Ambiente, seus fins e mecanismos de formulação e aplicação, e dá outras providências. Diário Oficial da União 1981; 02 set.

17. Brasil. Ministério do Meio Ambiente. Resolução CONAMA no 1/86, de 23 de janeiro de 1986. Dispõe sobre procedimentos relativos a Estudo de Impacto Ambiental. Diário Oficial da União 1986; 02 maio.

18. Brasil. Ministério do Meio Ambiente. Resolução CONAMA no 237/97, de 19 de dezembro de 1997. Diário Oficial da União 1997; 19 dez.

19. Mariano JB. Impactos ambientais do refino de petróleo [dissertação]. Rio de Janeiro: Universidade Federal do Rio de Janeiro; 2001.

20. Pedrozo MF, Barbosa EM, Corseuil HX, Schneider MR, Linhares MM. Ecotoxicologia e Avaliação de Risco do Petróleo. Salvador: Centro de Recursos Ambientais; 2002. 229 p.

21. Epstein PR, Selber J, organizadores. Oil: A life cycle analysis of its health and environmental impacts. Boston: The Center for Health and the Global Environment, Harvard Medical School; 2002.

22. Brasil. Ministério do Meio Ambiente. Resolução CONAMA no 23/1994, de 7 de dezembro de 1994. Diário Oficial da União 1994; $30 \mathrm{dez}$.

23. Brasil. Ministério do Meio Ambiente. Resolução CONAMA no 350/2004, de 6 de julho de 2004. Dispõe sobre o licenciamento ambiental específico das atividades de aquisição de dados sísmicos marítimos e em zonas de transição. Diário Oficial da União 2004; 20 ago.

24. Machado EA. Cidades Saudáveis: Relacionando Vigilância em Saúde e Licenciamento Ambiental através da Geografia [dissertação]. Brasília: Universidade de Brasília; 2007.

25. Hacon S, Shultz G, Bermejo PM. Indicadores de Saúde Ambiental: uma Ferramenta para a Gestão Integrada de Saúde e Ambiente. Cad Saude Colet 2005, 13(1):45-66.

26. Netto GF, Carneiro FF. Vigilância Ambiental em Saúde no Brasil. Ciência \& Ambiente 2002; 25:47-58.

27. Brasil. Ministério da Saúde (MS). Secretaria de Vigilância em Saúde. Coordenação Geral de Vigilância Ambiental em Saúde. Relatório do $7^{\circ}$ Seminário de Áreas Contaminadas e Saúde. Brasília-DF: Ministério da Saúde (MS), Secretaria de Vigilância em Saúde (SVS), Coordenação Geral de Vigilância em Saúde Ambiental (CGVAM); 2008.

28. Brasil. Ministério do Meio Ambiente. Resolução CONAMA no 286/2001, de 30 de agosto de 2001. Dispõe sobre o licenciamento de empreendimentos nas regiões endêmicas de malária. Diário Oficial da União 2001; 17 dez.

29. Minayo MCS. O desafio do conhecimento: pesquisa qualitativa em saúde. 10a ed. São Paulo: Hucitec; 2007. 
30. Brasil. Instituto Brasileiro do Meio Ambiente e Recursos Naturais Renováveis (IBAMA). Sistema Informatizado de Licenciamento Ambiental Federal (SISLIC). [Documento na Internet]. [acessado $2009 \mathrm{dez}$ 21]. Disponível em: http://www.ibama.gov.br/ licenciamento.

31. Rede Interagencial de Informações para a Saúde (RIPSA). Indicadores básicos para a saúde no Brasil: conceitos e aplicações. Brasília: Organização Panamericana de Saúde (OPAS); 2002.

32. Sánchez AIM, Bertolozzi MR. Pode o conceito de vulnerabilidade apoiar a construção do conhecimento em Saúde Coletiva? Cien Saude Colet 2007; 12(2):319-324.

Artigo apresentado em 11/03/2010 Aprovado em 30/07/2010

Versão final apresentada em 18/08/2010 